\title{
La dureza del agua como indicador básico de la presencia de incrustaciones en instalaciones domésticas sanitarias
}

\section{Water Hardness as Basic Indicator the Presence Fouling in Domestic Sanitary Facilities}

\author{
Julián-Soto F. \\ Centro Interdisciplinario de Investigación para el Desarrollo Integral Regional. \\ Unidad-Oaxaca. \\ CIIDIR-Instituto Politécnico Nacional-Oaxaca. \\ E-mail:julian_oaxmx@hotmail.com
}

(Recibido: octubre de 2007; reevaluado: abril de 2009; aceptado: septiembre de 2009)

\section{Resumen}

Se hizo el monitoreo de la calidad de agua en los valles centrales del estado de Oaxaca encontrando concentraciones muy elevadas de calcio, magnesio y carbonatos en las aguas provenientes de pozos profundos que se ubican en la periferia del río Atoyac, a diferencia del agua que proviene de las partes altas del valle de Etla, donde se tienen durezas máximas de $140 \mathrm{ppm}$, por lo que para la distribución de ambos efluentes a la ciudad de Oaxaca y Municipios conurbados, se propone hacer una dilución en la planta potabilizadora del Fortín, en la misma ciudad de Oaxaca; ya que después de determinar los índices de Langelier y otros, en cada una de las fuentes de abastecimiento con software localizados en la bibliografía universal, encontramos que la corriente proveniente de las riveras del río Atoyac, tiene propiedades incrustantes y corrosivas. Haciendo las pruebas de laboratorio para encontrar la mejor dilución que fue de 60 a $40 \%$ en volumen del gasto proveniente del Valle de Etla y de la periferia del río respectivamente, el índice de Langelier se transformó a un valor negativo, indicando que está subsaturada con respecto al carbonato de calcio $\left(\mathrm{CaCO}_{3}\right)$ y que tenderá a disolverse, evitando con estas acciones las incrustaciones que deterioran las unidades sanitarias.

Descriptores: agua, dilución, incrustante, dureza.

Abstract
The monitoring of the water quality was done in the central valleys of Oaxaca state, find-
ing very high concentrations of calcium, magnesium and carbonate in the waters from of
deep wells that are located in the periphery of the river Atoyac, unlike the water that comes
from the high parts of Etla valley, where there are maximum hardnesses of 140ppm, so for
the distribution of both effluent to the city of Oaxaca and neighboring municipalities, sug-
gesting a further dilution of the water treatment plant in "Fortin" in the city of Oaxaca,
that after determining the Langelier index and other, with software found in the universal
bibliography. The results indicate that water from the river Atoyac, have corrosive proper-
ties. In laboratory doing tests, the best dilution was 60 to $40 \%$ by volume, of water from


DOI: http://dx.doi.org/10.22201/fi.25940732e.2010.11n2.014

La dureza del agua como indicador básico de la presencia de incrustaciones en instalaciones domésticas sanitarias

Etla Valley and the periphery of the river respectively. Langelier index was converted to a negative, indicating saturation with respect to calcium carbonate ( $\mathrm{CaCO} 3)$ and will tend to dissolve, avoiding the deterioration of the sanitary units.

Keywords: Water, dilution, incrusting, hardness.

\section{Introducción}

Las aguas naturales contienen diversas cantidades de sales de composición variada y tienen la particularidad que al utilizarlas para el lavado con jabones de elevado peso molecular se forman precipitados en forma de grumos ésto se debe más que nada a sales solubles de calcio y magnesio. La dureza puede ser temporal o permanente, en el primer caso, el agua puede contener bicarbonato de calcio y de magnesio, hierro o magnesio. Se caracteriza por que su ablandamiento se logra con la ebullición, que consiste en que el bicarbonato se precipita desprendiendo dióxido de carbono y disminuyendo el valor del pH por las formaciones de ácido carbónico como se indica en las siguientes ecuaciones (Babor, 1996):

$$
\begin{aligned}
& \mathrm{Ca}^{+2}+2 \mathrm{HCO}_{3}^{-} \stackrel{\Delta}{\rightarrow} \mathrm{CaCO}_{3}+\mathrm{H}_{2} \mathrm{CO}_{3} \\
& \mathrm{H}_{2} \mathrm{CO}_{3} \stackrel{\Delta}{\rightarrow} \mathrm{H}_{2} \mathrm{O}+\mathrm{CO}_{3}
\end{aligned}
$$

Sin embargo, resulta más práctico y económico agregar cal apagada $\mathrm{Ca}(\mathrm{OH})_{2}$ en cantidades elevadas para reaccionar con los bicarbonatos, precipitándose los carbonatos correspondientes (Jairo, 2002) como indican la ecuaciones siguientes:

$$
\begin{aligned}
& \mathrm{Ca}^{+2}+2 \mathrm{HCO}_{3}^{-}+\mathrm{Ca}^{+2}+2 \mathrm{OH} \rightarrow 2 \mathrm{CaCO}_{3} \downarrow+2 \mathrm{H}_{2} \mathrm{O} \\
& \mathrm{Fe}^{+2}+2 \mathrm{HCO}_{3}+\mathrm{Ca}^{+2}+2 \mathrm{OH} \rightarrow \mathrm{CaCO}_{3} \downarrow+ \\
& \mathrm{FeCO}_{3} \downarrow+2 \mathrm{H}_{2} \mathrm{O}
\end{aligned}
$$

La dureza permanente es producida por sales más fijas como los cloruros $\left(\mathrm{Cl}^{-}\right)$, sulfatos $\left(\mathrm{SO}^{2-}{ }_{4}\right)$, encontrando que la dureza total es la suma con la dureza temporal y que se acostumbra representarla en función de los bicarbonatos de calcio y de magnesio (Babor, 1996).

Como se mencionó anteriormente, la dureza es causada por iones metálicos divalentes que bien pueden ser calcio $\left(\mathrm{Ca}^{2+}\right)$, magnesio $\left(\mathrm{Mg}^{2+}\right)$, estroncio $\left(\mathrm{Sr}^{2+}\right)$, fierro $\left(\mathrm{Fe}^{2+}\right)$ y aniones como bicarbonato $\left(\mathrm{HCO}_{3}^{-}\right)$, sulfatos $\left(\mathrm{SO}_{4}{ }^{2-}\right)$, cloruros $\left(\mathrm{Cl}^{-}\right)$, nitratos $\left(\mathrm{NO}_{3}^{-}\right)$y silicatos (Jairo, 2002).

Las aguas que contienen mayor cantidad de bicarbonatos de calcio y de magnesio, representa la principal forma de alcalinidad, y en algunos casos, es equivalente a la dureza carbonatada o total, que es igual a la temporal, o que produce incrustaciones o almacenamientos leves que se pueden remover mediante inyecciones de aire o agua a presión (Gomella, 1999).

También se tiene la dureza no carbonatada que se define como la diferencia entre la dureza total y la alcalinidad; que son compuestos carbonatados que se transforman a cristales del elemento correspondiente por la elevación de la temperatura, es un material duro y resistente que se almacena en las paredes internas de las tuberías y accesorios de calentadores y calderas, habiendo una pérdida en la transferencia de calor (Vernon, 2002).

Durante las diversas reacciones químicas, que se forman durante las incrustaciones, es importante analizar la aplicación de los principios de la termodinámica en equilibrio con la química del agua para estimar la tendencia del bicarbonato de calcio a precipitarse o a disolverse, considerando siempre un sistema cerrado, donde no se podrá adicionar ni eliminar materia con la fluidez de la energía, operando bajo condiciones de presión y temperatura constantes, como se indica en las ecuaciones $(1,2,3,4,5,6,7,8,9,10,11)$.

$$
G=H-T S
$$

La expresión matemática anterior (1) representa la manera de evaluar la energía libre de Gibbs $(G)$ kcal, en función de la entalpía (H) kcal, que es el contenido total de energía de un elemento o compuesto, (T) es la temperatura a la cual se realiza el proceso y $(\mathrm{S})$ es la entropía que de alguna manera es la energía molecular interna, siendo el producto (TS) la parte de la energía total que no está disponible para realizar trabajo útil.

Para el caso de una reacción en equilibrio, la energía libre del sistema será mínima y se tiene.

$$
A A+b B \leftrightarrow c C+d D
$$

Lo cual indica que al agregar la sustancia $\mathrm{A}$ y $\mathrm{B}$ a un recipiente la energía libre total (GT) será igual a la suma de las energías libres de cada componente en la reacción química, entonces:

$$
G T=n A \bar{G} A+n B \bar{G} B+n C \bar{G} C+n D \bar{G} D
$$


Esto significa que a medida de cualquier reacción se realiza en un tiempo cualquiera la condición de equilibrio de una reacción, ya que tiene su mínimo valor de energía libre y es proporcional a $\Delta \mathrm{G}$, que se define:

$$
\Delta G=\left(\sum \gamma_{i} \bar{G}_{i}\right)_{\text {productos }}-\left(\sum \gamma_{i} \bar{G}_{i}\right)_{\text {reac tan tes }}
$$

Considerando $(\gamma i)$ el coeficiente estequiométrico que indica la cantidad de energía necesaria, para el desarrollo del procesos y ( $\mathrm{G} i$ ) en la energía libre (moles) (Vernon, 2002); siendo la expresión matemática para calcular $(\Delta \mathrm{G})$ en cualquier proceso químico:

$$
\begin{aligned}
& \Delta G=\Delta G^{0}+R T \ln \frac{(C)^{c}(D)^{d}}{(A)^{a}(B)^{b}} \\
& \text { pero } \Delta G^{0}=\left(\sum \gamma_{i} \bar{G}_{0}\right)_{\text {productos }}-\left(\sum \gamma_{i} \bar{G}_{0}\right)_{\text {reactantes }}
\end{aligned}
$$

Que para el caso del carbonato de calcio, se tendrá:

$$
\begin{aligned}
& H^{+}+\mathrm{CO}_{3}^{-} \rightarrow \mathrm{CaCO}_{3}^{-} \\
& \Delta G=\Delta G^{0}+R T 1 n Q \\
& \Delta G^{0}=-R T 1 n K \\
& Q=\frac{\left\{\mathrm{Ca}^{+2}\right\}\left\{H C O_{3}^{-1}\right\}}{\left\{H^{+1}\right\}} \\
& p H=-\log \left[H^{+}\right]
\end{aligned}
$$

En el caso de tener $\Delta G>0$, se tendrá precipitación de $\mathrm{CaCO}_{3}$. Otra forma de evaluar las características incrustantes o corrosivas es por medio del índice de Langelier que se define como la diferencia entre el $\mathrm{pH}$ real del agua y el $\mathrm{pH}$ hipotético, si ésta estuviera en equilibrio con $\mathrm{CaCO}_{3}$ (Vernon, 2002)

$$
\text { I. } L=p H_{a}-p H_{s}
$$

Que indica que si $(\mathrm{IL}=\mathrm{O})$ el agua estará en equilibrio con $\mathrm{CaCO}_{3}$, si $(\mathrm{IL}=+)$ el agua estará sobre saturada con respecto a $\mathrm{CaCO}_{3}$ y tenderá a precipitarse; finalmente, si $(\mathrm{IL}=-)$, el agua estará subsaturada con respecto a $\mathrm{CaCO}_{3}$ y el contaminante tenderá a disolverse (Vernon, 2002).

\section{Metodología}

Metcalf y Eddy (1996) realizaron trabajos de dilución de corrientes para remover y disminuir la concentración de contaminantes; para este trabajo se instalaron dos recipientes de 19 litros, de donde se extrajo agua de diferentes concentraciones en cuanto a la composición química, usando para este fin 2 bombas masterflex, de tal manera que con las válvulas de control instaladas en las salidas fue posible medir los gastos de cada una de las corrientes.

Posteriormente, se tomaron muestras de agua del depósito de almacenamiento hasta encontrar la mezcla óptima en cuanto a la menor dureza total del agua, se hicieron 16 corridas formando igual número de mezclas y midiendo la dureza total de dos maneras, con un kit Hach y con el método volumétrico E.D.T.A. los cuales son exclusivos para este fin.

Finalmente, se hicieron las interpretaciones adecuadas para el procesamiento de datos, considerando los resultados de laboratorio, tanto fisicoquímicos como de metales pesados.

\section{Ubicación del área de estudio}

El Río Atoyac brota de las partes altas del Valle de Etla, en un lugar denominado las Sodas, perteneciente al Municipio de San Francisco Telixtlahuaca, desciende por las poblaciones de San Pablo Huitzo y Villa de Etla, atravesando la ciudad de Oaxaca, llegando a Cuilápam de Guerrero, (lugar donde fue encarcelado y fusilado uno de los héroes de nuestra Independencia, Gral. Vicente Guerrero), continua su trayecto hasta desembocar en la Costa Oaxaqueña.

Desde el tramo de la Villa de Etla, hasta Cuilápam de Guerrero, se han realizado una serie de perforaciones de pozos profundos que abastecen de agua a las poblaciones aledañas, no siendo la excepción el Municipio de Oaxaca de Juárez y 18 municipios conurbados, que reciben el agua de abastecimiento público de la planta potabilizadora denominada El Fortín, la cual se alimenta de más de 20 pozos que se tienen en la periferia del río Atoyac en la ciudad de Oaxaca y que en el mapa (figura 1) se indica con la letra (A) ó número 67.

Por otro lado, la misma planta potabilizadora recibe otro afluente de un manantial que brota en el Cerro de la Guacamaya, pasando por los pueblos de San Agustín y San José Etla, llegando a la ciudad de Oaxaca como se aprecia en la misma representación esquemática. 


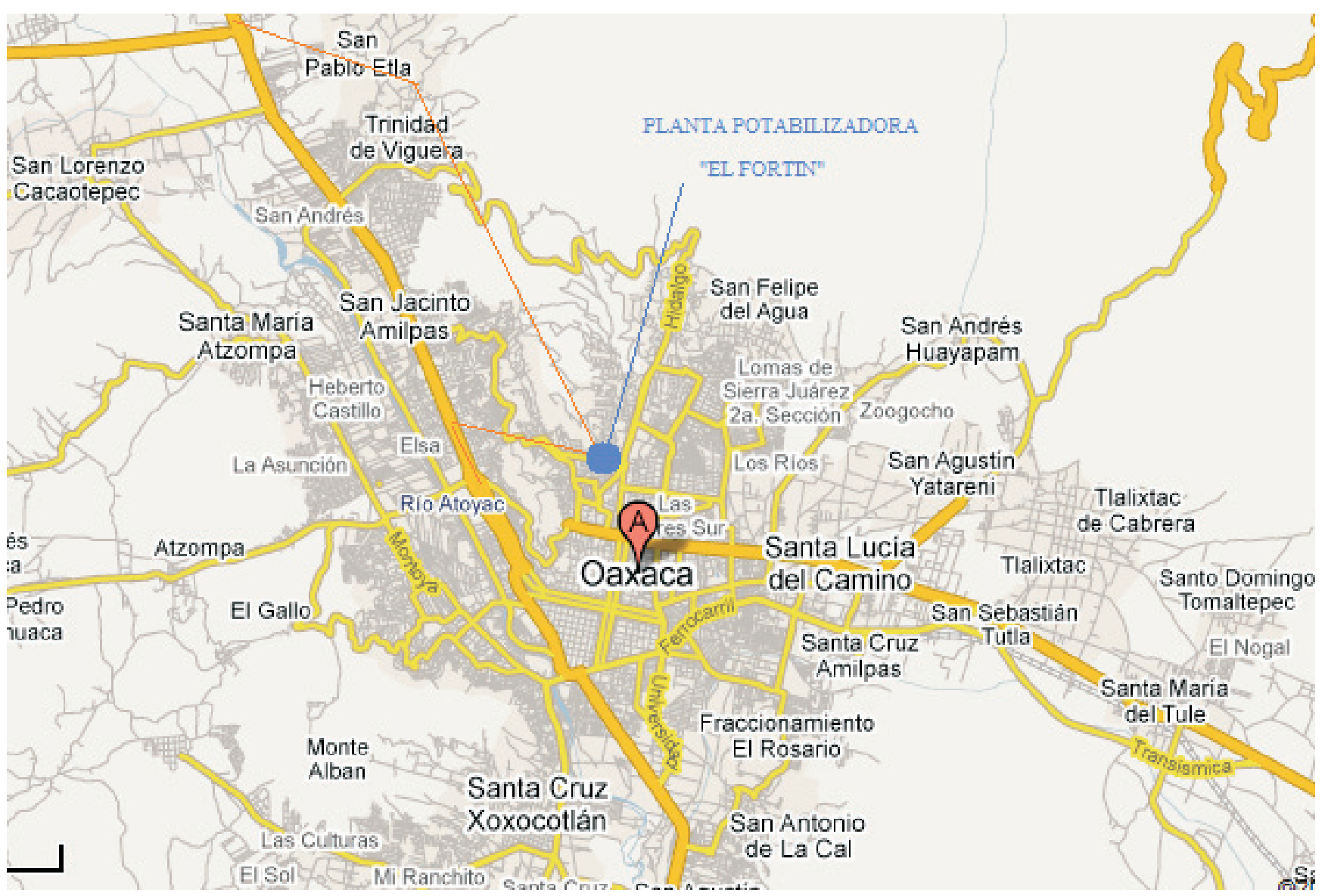

Figura 1. Mapa del área de estudio en el Valle de Oaxaca

\section{Parte experimental}

El presente trabajo de investigación tuvo como principal objetivo representar los porcentajes en volumen de los fluidos procedentes de la zona alta del valle de Etla y de los pozos que se ubican en la zona aledaña a la rivera del río Atoyac. Durante el experimento, se utilizaron dos bombas Masterflex que extraen el agua de dos garrafones que contienen agua de las fuentes mencionadas, controladas por una válvula en la salida con el fin de variar los gastos que llegaban a un depósito mezclador; de este último recipiente se tomaron las muestras de agua a diferentes volúmenes y se medía la dureza total con un kit, marca Hach, cuyos valores fueron confirmados en el laboratorio de CIIDIR-OAXACA por medio de la aplicación del método volumétrico E.D.T.A, hasta encontrar la mejor proporción. Con los resultados obtenidos de la caracterización completa del agua se ingresaron al software y se encontraron los resultados de los índices medidos de la mezcla óptima.

\section{Diseño del prototipo}

Se trató entonces de encontrar la mejor mezcla óptima de 2 corrientes de agua, la primera proveniente de la zona alta del Valle de Etla y que aproximadamente representa el 30\% del gasto total de agua en los Valles Centrales; y la otra corriente proveniente de los pozos profundos y que se estima de un gasto de $70 \%$ de un consumo total de $360,796 \mathrm{~m}^{3}$ / día para evitar la corrosión en tuberías y accesorios sanitarios de uso en casas habitación y otras; para tal fin, se pensó en utilizar como materiales: dos garrafones de 19 litros, dos bombas masterflex, cables de corriente eléctrica, recipiente de plástico, válvulas de control, tuberías, kit para dureza total, potenciómetro, bidones de (19 1) de PET para el muestreo y caracterización del agua.

En la figura 1 se tiene la ubicación de la zona alta de Etla, que por su topografía, llega a la Planta potabilizadora del Fortín, sin recibir ninguna energía más que la de la fuerza de gravedad, del mismo modo, se observa la 
zona baja de la periferia del río Atoyac, donde se utilizan sistemas de bombeo para enviar el preciado líquido al mismo sistema purificador.

Una vez armado el prototipo, se procedió a su operación, muestreo y caracterización del agua.

\section{Operación}

Las bombas masterflex succionan el agua de cada uno de los recipientes $(1,2)$, en las proporciones dosificadas por las válvulas $(4,7)$, tomando soluciones de diversa composición en (8); de este último recipiente se tomaron las diversas muestras en volúmenes diferentes, lo que se observaba en cada uno de los recipientes utilizados (figura 2), tomando las mezclas que se dan en la tabla 1.

\section{Procedimiento de operación}

Los depósitos (1) y (2) de la figura (2) se llenaron con las aguas provenientes de Etla y Río Atoyac, respectivamente, se regularon las salidas del agua con las válvulas (3) y (4), recibiendo la mezcla en el recipiente (8), midiendo los gastos con los recipientes originales, y haciendo un volumen de $3 \mathrm{~L}$ en cada una de las pruebas.



Figura 2. Esquema del prototipo para la realización de la mezcla óptima

Tabla 1. Volúmenes utilizados en la formación de la mezcla óptima

\begin{tabular}{cccccc}
\hline Experiencia & Parámetro & $\% 1$ vol. & $\% 2$ vol. & vol. total & Resultado \\
\hline 1 & Dureza total & $60(1.8) \mathrm{L}$ & $40(1.2) \mathrm{L}$ & $3 \mathrm{~L}$ & $320 \mathrm{mg} / \mathrm{L} \mathrm{CaCO}_{3}$ \\
2 & Dureza total & $50(1.5) \mathrm{L}$ & $50(1.5) \mathrm{L}$ & $3 \mathrm{~L}$ & $280 \mathrm{mg} / \mathrm{L} \mathrm{CaCO}_{3}$ \\
3 & Dureza total & $40(1.2) \mathrm{L}$ & $60(1.8) \mathrm{L}$ & $3 \mathrm{~L}$ & $220 \mathrm{mg} / \mathrm{L} \mathrm{CaCO}_{3}$ \\
4 & Dureza total & $30(0.9) \mathrm{L}$ & $70(2.1) \mathrm{L}$ & $3 \mathrm{~L}$ & $180 \mathrm{mg} / \mathrm{L} \mathrm{CaCO}_{3}$ \\
\hline
\end{tabular}


DOI: http://dx.doi.org/10.22201/fi.25940732e.2010.11n2.014

La dureza del agua como indicador básico de la presencia de incrustaciones en instalaciones domésticas sanitarias

Tabla 2. Composición química del agua proveniente de pozos profundos

\begin{tabular}{lcc}
\hline \multicolumn{1}{c}{ Parámetros } & Unidades & Resultado influente \\
\hline Dureza total & $\mathrm{mg} / \mathrm{L}\left(\mathrm{CaCO}_{3}\right)$ & 473.19 \\
Dureza al calcio & $\mathrm{mg} / \mathrm{L}\left(\mathrm{CaCO}_{3}\right)$ & 222.81 \\
Dureza al magnesio & $\mathrm{mg} / \mathrm{L}\left(\mathrm{CaCO}_{3}\right)$ & 247.64 \\
Magnesio libre & $\mathrm{mg} / \mathrm{L}$ & 60,31 \\
Calcio libre & $\mathrm{mg} / \mathrm{L}$ & 88.85 \\
Fierro & $\mathrm{mg} / \mathrm{L}$ & 1.16 \\
Manganeso & $\mathrm{mg} / \mathrm{L}$ & 2.09 \\
pH & & 7.6 \\
Turbiedad & $\mathrm{NTU}$ & 12.86 \\
Dureza de $\mathrm{CO}_{3}(\mathrm{mg} / \mathrm{L})$ & $\mathrm{mg} / \mathrm{L}(\mathrm{CaCO})_{3}$ & 242.95 \\
\hline
\end{tabular}

Tabla 3. Composición química del agua proveniente de manantiales de los Valles altos de Etla, Oax. (manantial)

\begin{tabular}{lcc}
\hline \multicolumn{1}{c}{ Parámetros } & Unidades & Resultado efluente \\
\hline Dureza total & $\mathrm{mg} / \mathrm{L}\left(\mathrm{CaCO}_{3}\right)$ & 140 \\
Dureza al calcio & $\mathrm{mg} / \mathrm{L}\left(\mathrm{CaCO}_{3}\right)$ & 80 \\
Dureza al magnesio & $\mathrm{mg} / \mathrm{L}\left(\mathrm{CaCO}_{3}\right)$ & 60 \\
Magnesio libre & $\mathrm{mg} / \mathrm{L}$ & 27 \\
Calcio libre & $\mathrm{mg} / \mathrm{L}$ & 39 \\
Fierro & $\mathrm{mg} / \mathrm{L}$ & 0.86 \\
Manganeso & $\mathrm{mg} / \mathrm{L}$ & 1.7 \\
pH & & 7.4 \\
Turbiedad & $\mathrm{NTU}$ & 10.3 \\
Dureza de $\mathrm{CO}_{3}(\mathrm{mg} / \mathrm{L})$ & $\mathrm{mg} / \mathrm{L}(\mathrm{CaCO})$ & 136.0 \\
\hline
\end{tabular}

En estudios realizados se hace una clasificación del agua conforme la dureza total, como se muestra en la tabla (4) (Jairo, 2002).
La composición química del agua de pozos profundos (tabla 2) es muy dura, por lo que se tiene un índice de Langelier positivo, como se indica en la tabla 5.

Tabla 4. Concentraciones estandars para definir las características del agua

\begin{tabular}{cc}
\hline Concentración $(\mathrm{Ca}+\mathrm{Mg})$ & Características \\
\hline $0-75 \mathrm{mg} / \mathrm{L}$ & Blanda \\
$75-200 \mathrm{mg} / \mathrm{L}$ & Moderadamente dura \\
$200-300 \mathrm{mg} / \mathrm{L}$ & Dura \\
$>300 \mathrm{mg} / \mathrm{L}$ & Muy dura \\
\hline
\end{tabular}


DOI: http://dx.doi.org/10.22201/fi.25940732e.2010.11n2.014

Julián-Soto $\mathrm{F}$.

Tabla 5. Software de cálculo de Índice de Langelier y otros

\begin{tabular}{|c|c|c|}
\hline Parámetro & Unidades & Valor \\
\hline $\mathrm{pH}$ & & 7.6 \\
\hline C.E & $\mu \mathrm{S} / \mathrm{cm}$ & 880 \\
\hline TDS & $\mathrm{mg} / \mathrm{L}$ & 563 \\
\hline $\mathrm{Ca}^{+2}$ & $\mathrm{mg} / \mathrm{L}$ & 88.85 \\
\hline $\mathrm{Ca}^{+2}$ & $\mathrm{mg} / \mathrm{L} \mathrm{CaCO}_{3}$ & 222.81 \\
\hline $\mathrm{HCO}_{3}^{-}$ & $\mathrm{mg} / \mathrm{L}$ & 178.1 \\
\hline $\mathrm{CO}_{3}^{2-}$ & $\mathrm{mg} / \mathrm{L}$ & 0 \\
\hline * Alcalinidad & $\mathrm{mg} / \mathrm{L} \mathrm{CaCO}_{3}$ & 146.0 \\
\hline A & & 0.18 \\
\hline B & & 1.97 \\
\hline $\mathrm{C}$ & & 1.95 \\
\hline $\mathrm{D}$ & & 2.16 \\
\hline phsaturación & & 7.33 \\
\hline pHeq & & 7.84 \\
\hline $\mathrm{T}^{\mathrm{a}}$ & ${ }^{\circ} \mathrm{C}$ & 21 \\
\hline Langelier (LSI) & & 0.3 \\
\hline Ryznar (RSI) & & 7.1 \\
\hline Puckorius (PSI) & & 6.8 \\
\hline
\end{tabular}

Sin embargo, con la caracterización realizada al agua de manantial el I.L. (Índice de Langelier) es negativo, por lo cual el $\mathrm{CaCO}_{3}$ tiende a disolverse. Estas características nos permiten proponer una dilución de los influentes a la planta potabilizadora, que abastece de agua a la ciudad de Oaxaca (tabla 6).

Tabla 6. Software de cálculo de Índice de Langelier y otros (continúa ...)

\begin{tabular}{ccc}
\hline Parametro & Unidades & Valor \\
\hline $\mathrm{pH}$ & & 7.4 \\
$\mathrm{C} . \mathrm{E}$ & $\mu \mathrm{S} / \mathrm{cm}$ & 880 \\
$\mathrm{TDS}$ & $\mathrm{mg} / \mathrm{L}$ & 563 \\
$\mathrm{Ca}^{+2}$ & $\mathrm{mg} / \mathrm{L}$ & 39 \\
$\mathrm{Ca}^{+2}$ & $\mathrm{mg} / \mathrm{L} \mathrm{CaCO}{ }_{3}$ & 80.00 \\
$\mathrm{HCO}_{3}^{-}$ & $\mathrm{mg} / \mathrm{L}$ & 178.1 \\
$\mathrm{CO}_{3}^{2-}$ & $\mathrm{mg} / \mathrm{L}$ & 0 \\
$* \mathrm{Alcalinidad}^{-}$ & $\mathrm{mg} / \mathrm{L} \mathrm{CaCO}$ & 146.0 \\
$\mathrm{~A}$ & & \\
$\mathrm{~B}$ & & 0.18 \\
$\mathrm{C}$ & & 1.97 \\
$\mathrm{D}$ & & 1.50 \\
\end{tabular}


DOI: http://dx.doi.org/10.22201/fi.25940732e.2010.11n2.014

La dureza del agua como indicador básico de la presencia de incrustaciones en instalaciones domésticas sanitarias

Tabla 6. Software de cálculo de Índice de Langelier y otros (... continuación)

\begin{tabular}{ccc}
\hline Parametro & Unidades & Valor \\
\hline phsaturación & & 7.78 \\
pHeq & 7.84 \\
& & \\
$\mathrm{~T}^{\mathrm{a}}$ & ${ }^{\circ} \mathrm{C}$ & 21 \\
& & \\
Langelier (LSI) & -0.4 \\
Ryznar (RSI) & 8.2 \\
Puckorius (PSI) & 7.7 \\
\hline
\end{tabular}

\section{Resultados}

La medición de dureza total como $\mathrm{CaCO}_{3}(\mathrm{mg} / \mathrm{L})$, en la mezcla óptima encontrada se muestra en la tabla 7.

En la gráfica de la figura 3 se indica, la concentración de la dureza total del agua, con respecto a la mezcla óptima encontrada.

Tabla 7. Concentraciones de dureza total preliminar a la caracterización final del agua

\begin{tabular}{ccc}
\hline FA (\%) & FB(\%) & Concentración ppm \\
\hline 20 & 80 & 80 \\
30 & 70 & 120 \\
40 & 60 & 140 \\
50 & 50 & 190 \\
60 & 40 & 240 \\
70 & 30 & 260 \\
80 & 20 & 330 \\
\hline
\end{tabular}

Experiencia (1) muestra de 1L ( FA = muestra manantial, FB = muestra pozos)

\begin{tabular}{ccc}
\hline FA $(\%)$ & $\mathrm{FB}(\%)$ & $\mathrm{VT}(\mathrm{ml})$ \\
\hline 20 & 80 & 1000 \\
30 & 70 & 1000 \\
40 & 60 & 1000 \\
50 & 50 & 1000 \\
60 & 40 & 1000 \\
70 & 30 & 1000 \\
80 & 20 & 1000 \\
\hline
\end{tabular}




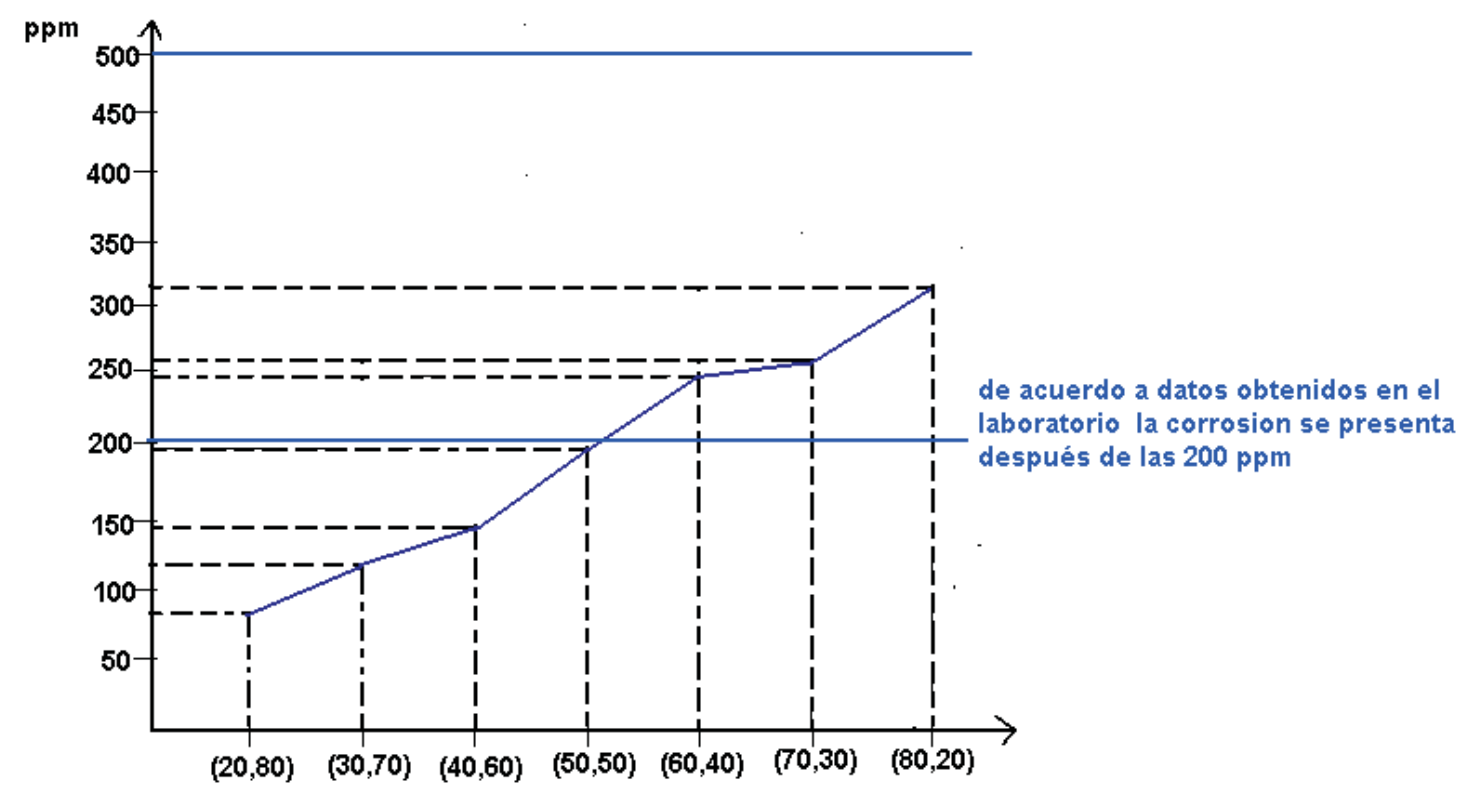

Figura 3. Representación de la mezcla óptima con las propiedades corrosivas o incrustantes del agua

De esta manera encontramos que la mejor proporción es la 60 y 40\% de agua, que de un volumen de 1L, $400 \mathrm{~mL}$ correspondieron al agua de pozo y $600 \mathrm{~mL}$ de agua de manantial para formar una mezcla óptima, con una dureza total de 140 a 160 ppm, cuyos parámetros finales se resumen en la tabla 8.
En la tabla 9 se marca la determinación del índice de Langelier, con un valor de (-0.2), que corresponde a la composición química del agua mezclada con las 2 corrientes manejadas.

Tabla 8. Composición química del agua mezclada en una relación 60 y 40\%

\begin{tabular}{cccc}
\hline Parámetro & Unidades & Resultado & NOM-127-SSA-1996 \\
\hline Dureza total & $\mathrm{mg} / \mathrm{L}\left(\mathrm{CaCO}_{3}\right)$ & 160 & 500 \\
Dureza Ca+2 & $\mathrm{mg} / \mathrm{L}\left(\mathrm{CaCO}_{3}\right)$ & 90 & 300 \\
Dureza al Mg+2 & $\mathrm{mg} / \mathrm{L}\left(\mathrm{CaCO}_{3}\right)$ & 70 & 200 \\
Magnesio libre & $\mathrm{mg} / \mathrm{L}$ & 42 & - \\
Calcio libre & $\mathrm{mg} / \mathrm{L}$ & 38 & - \\
Fierro & $\mathrm{mg} / \mathrm{L}$ & 0.05 & 0.30 \\
Manganeso & $\mathrm{mg} / \mathrm{L}$ & 1.9 & 0.15 \\
pH & & 7.5 & $6.5-8.5$ \\
Turbiedad & $\mathrm{NTU}$ & 5.5 & $5(\mathrm{NTU})$ \\
Dureza de $\mathrm{CO}_{3}$ & $\left.\mathrm{mg} / \mathrm{L}(\mathrm{CaCO})_{3}\right)$ & 120 & 300 \\
\hline
\end{tabular}


DOI: http://dx.doi.org/10.22201/fi.25940732e.2010.11n2.014

La dureza del agua como indicador básico de la presencia de incrustaciones en instalaciones domésticas sanitarias

Tabla 9. Software de cálculo de Índice de Langelier y otros

\begin{tabular}{|c|c|c|}
\hline Parámetro & Unidades & Valor \\
\hline $\mathrm{pH}$ & $\mu \mathrm{S} / \mathrm{cm}$ & 7.5 \\
\hline C.E & $\mathrm{mg} / \mathrm{L}$ & 880 \\
\hline TDS & $\mathrm{mg} / \mathrm{L}$ & 563 \\
\hline $\mathrm{Ca}^{+2}$ & $\mathrm{mg} / \mathrm{L}$ & 38 \\
\hline $\mathrm{Ca}^{+2}$ & $\mathrm{mg} / \mathrm{L} \mathrm{CaCO}_{3}$ & 90.00 \\
\hline $\mathrm{HCO}_{3}^{-}$ & $\mathrm{mg} / \mathrm{L}$ & 178.1 \\
\hline $\mathrm{CO}_{3}^{2-}$ & $\mathrm{mg} / \mathrm{L}$ & 0 \\
\hline * Alcalinidad & $\mathrm{mg} / \mathrm{L} \mathrm{CaCO} 3$ & 146.0 \\
\hline A & & 0.18 \\
\hline B & & 1.97 \\
\hline $\mathrm{C}$ & & 1.55 \\
\hline $\mathrm{D}$ & & 2.16 \\
\hline phsaturación & & 7.72 \\
\hline pHeq & & 7.84 \\
\hline $\mathrm{T}^{\mathrm{a}}$ & ${ }^{\circ} \mathrm{C}$ & 21 \\
\hline Langelier (LSI) & & -0.2 \\
\hline Ryznar (RSI) & & 7.9 \\
\hline Puckorius (PSI) & & 7.6 \\
\hline
\end{tabular}

\section{Análisis de resultados}

Los efectos de las sales que contiene el agua proveniente de los pozos profundos en los valles centrales de Oaxa$\mathrm{ca}$, son adversos en las instalaciones sanitarias, que se manifiestan por el almacenamiento de incrustaciones. Esta observación se confirma con el índice de Langelier que tuvo un valor de 0.3; a diferencia del agua que proviene de manantiales donde se tienen durezas bajas y cuyo índice de Langelier es de -0.4 , indicando con este valor la disociación de las sales, y por tanto, evitando con esto la corrosión en instalaciones domésticas, que solamente tienen un periodo de vida menor a 12 meses.

El fenómeno de dilución, tal y como lo proponen Metcalf (1996) en el tema de dotación de agua, ayuda a resolver problemas en comunidades con escasos recursos requeridos para el tratamiento y purificación del agua.

Después de haber realizado las diluciones, se encontró una combinación óptima de $40 \%$ del volumen de agua de pozos y $60 \%$ del volumen de agua de manantial; con una dureza de $160 \mathrm{mg} / \mathrm{L}$ como Carbonato de Calcio (tabla 8), disminuyendo todas las concentraciones de otros componentes y disminuyendo el Índice de Langelier a (-0.2), lo que garantiza una disociación de sales para evitar la destrucción de llaves hidráulicas, depósitos de agua en las tasas de baños, regaderas, válvulas de control, de agua, etc. Otros parámetros como Fierro $(\mathrm{Fe})$, Manganeso $(\mathrm{Mn})$, Turbiedad, tienen valores que rebasan los límites máximos permisibles que se encuentran en la NOM-127-SSA-1996, pero que no tienen efectos en las unidades sanitarias. 
La metodología de evaluación aplicada es bastante práctica utilizando el Software que recomienda la Secretaría Mundial de la Salud (2002) y que para la industria pudiera ser muy importante.

\section{Conclusiones}

La propuesta del método de dilución resulta viable y económica si se considera que las dos corrientes de agua están llegando a la planta potabilizadora denominada el fortín; por lo cual, solamente se requiere instalar válvula de control para la dosificación de los gastos de agua, formando la mezcla recomendada (con una relación de 40 a $60 \%$ en volumen).

Esta actividad permitirá dar un período de vida más largo en las unidades sanitarias que para el caso de las regaderas. En la actualidad, se deben cambiar por lo menos 3 veces por año, a diferencia de lo acostumbrado en el Distrito Federal, donde casi no se cambian.

Este estudio pretende ser una herramienta para apoyar a las autoridades de nuestro país en la evaluación de las características, corrosión o incrustantes del agua que se utilizan para el abastecimiento público, ya que afortunadamente en la actualidad los propietarios que pagan por un servicio de abastecimiento del agua, todavía no cobran el deterioro que reciben las unidades sanitarias y aceptan pasivamente cualquier calidad de agua.

Es importante comentar que el diseño del experimento es muy elemental, ya que en la actualidad no se tienen recursos económicos para el desarrollo de grandes proyectos; por lo que se busca una inversión de bajo costo para resolver grandes problemas de índole social y económico.

\section{Agradecimientos}

Reconozco sinceramente al Instituto Politécnico Nacional de nuestro país los apoyos recibidos, donde se incluyen los salarios percibidos y que entendemos debe ser un enorme sacrificio para el sostenimiento de una institución tan importante como es el CIIDIR-OAXACA. Asimismo, agradezco a los alumnos del Instituto Tecnológico de Oaxaca (ITO) su decidido apoyo, para el intercambio de opiniones y la captura del presente trabajo.

\section{Referencias}

Babor. Química general. Editorial Limusa. 1996.

Jairo A. Calidad del agua. Editorial Alfa Omega. 2a edición. 2002.

Gomella H. Tratamiento de aguas para abastecimiento público. Editores técnicos asociados, S.A. 1999.

Metcalf y Eddy. Ingeniería sanitaria, tratamiento, evacuación y reutilización de aguas residuales. Ed. Porrua. 1996.

Vernon J. Química del agua. Editorial Limusa. 2002.

\section{Bibliografía sugerida}

APHA,AWWA,WPCF. Standard Methods for the Examination of Water and Wastewater. 2002.

Departamento de Sanidad del Estado de Nueva York. Manual de Tratamiento de Aguas de abastecimiento. Ed. Limusa. 1998.

Jairo A. Potabilización del agua. Ed. Alfa Omega. $3^{a}$. Edición. 2001.

Linvil G.R. Operaciones unitarias de ingeniería sanitaria. Ed. Continental. 1998.

Norma Oficial Mexicana, NOM-127-SSA 1994.

Soto J. Manejo de las aguas residuales. Ed. Kalpulli. México. 2001.

\section{Semblanza del autor}

Félix Julián-Soto. Es ingeniero químico industrial egresado de la ESIOIE del Instituto Politécnico Nacional. Es profesor de matemáticas en la Escuela Normal Superior de México y maestro en ciencias en ingeniería química del Instituto Tecnológico de Oaxaca. Laboró en las Escuelas Secundarias Técnicas y Diurnas de DF. Actualmente se encuentra en el Centro de Investigación, denominado (CIIDIR-OAXACA), perteneciente al mismo Politécnico Nacional de México, ha participado y dirigido proyectos de investigación relacionados con la calidad y tratamiento del agua potable y residuales. 